Z. klin. Chem. u. klin. Biochem.

8. Jg., S. $60-64$, Januar 1970

\title{
Untersuchung der Bindung von Schilddrüsenhormonen an Humanserum-Lipoproteine
}

\author{
Von G. Kostner und I. Abou Ex Eisch \\ Aus.dem Institut für physiologische Chemie der Universität Graz (Vorstand: Prof. Dr. A. Holasek)
}

(Eingegangen am 25. September 1969)

Zur Untersuchung der Bindungsfähigkeit der Humanserumlipoproteine für Schilddrüsenhormone wurden very low density lipoproteins (VLDL), low density lipoproteins (LDL) und high density lipoproteins (HDL) mit Hilfe spezifischer Antikörper bzw. einer polymeren Kieselsäure (Aerosil) aus dem Serum entfernt und die Verteilung der Hormone bestimmt. Dies erfolgte einerseits durch Bestimmung des eiweißgebundenen Jods, andererseits durch Messung der Radioaktivität von in vivo und in vitro markierten Seren. Dabei wurde gefunden, daß keines der erwähnten Lipoproteine im Gemisch mit den übrigen Serumproteinen nennenswerte Mengen an Schilddrüsen-

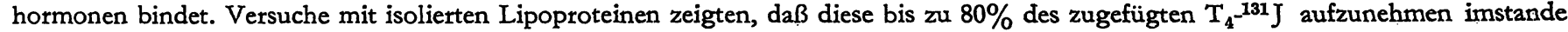
sind.

\section{Studies on the binding of thyroid bormones to buman serum lipoproteins}

In order to determine the binding between human serum lipoproteins and thyroid hormones, very low density lipoproteins (VLDL), low density lipoproteins (LDL) and high density lipoproteins (HDL) were separated from the serum with the aid of specific antibodies or a polymeric silicic acid (Aerosil). The distribution of the hormones was then determined by measurement of the protein-bound iodine and measurement of the radioactivity of in vivo and in vitro labelled sera. Negligible amounts of thyroid hormones were bound by the above lipoproteins in the presence of the other serum proteins. Experiments with isolated lipoproteins showed that these are able to bind up to $80 \%$ of the added $\mathrm{T}_{4^{-1}}^{-131} \mathrm{~J}$.

Die exogenen Schilddrüsenhormone werden zum größten Teil von 3 Serumproteinen, nämlich Präalbumin (TBPA), Albumin (TBA) und dem thyroxinbindenden Globulin (TBG) transportiert $(1,2,3,4,5)$.

Da mit Hilfe der Immunoelektrophorese und anschlieBender Autoradiographie von Humanseren, die radioaktives Thyroxin ( $\left.T_{4}\right)$ enthielten, Aktivität in den Lipoproteinbanden gefunden wurde, gelten auch diese als spezifische Thyroxinträger. So konnte K. Mryar (6) neben TBPA, TBA und TBG eine Bindung von $\mathrm{T}_{4}$ an $\alpha_{1}$ - und prae- $\beta$-Lipoproteine feststellen. Auch von anderen Arbeitsgruppen wird den Lipoproteinen eine Trägerfunktion für Schilddrüsenhormone zugeschrieben $(7,8$, $9,10,11)$. Gelegentlich sind noch andere, zum Teil unbekannte thyroxinbindende Proteine beschrieben worden $(5,12,13)$.

Die Bindung und quantitative Verteilung der Schilddrüsenhormone zwischen den einzelnen thyroxinbindenden Proteinen ist stark vom pH-Wert (14) sowie der Art und Konzentration von Fremdstoffen abhängig $(3,5$, $15,16,17)$. Aus diesem Grunde konnte die Rolle des TBPA als $\mathrm{T}_{4}$-bindendes Protein erst relativ spät entdeckt werden (18).

$\mathrm{Da}$ reine Lipoproteine aus dem Serum nach den herkömmlichen Methoden nur unter beträchtlicher Veränderung des Milieus (Erhöhung der Salzkonzentration und anschließendes Ultrazentrifugieren bzw. Zusatz von Salzen und selektiven Fällungsmitteln) gewonnen werden können, erschien es nicht sinnvoll, eine Isolierung nach diesen Methoden durchzuführen und anschließend die darin befindliche Menge $\mathrm{T}_{4}$ zu bestimmen. Hingegen erlaubt die Spezifität der Antigen-Antikörper-Reaktion in kürzester Zeit und ohne Veränderung des $\mathrm{pH}$-Wertes und der Salzkonzentration, alle Proteine aus einem Gemisch zu entfernen und anschließend $z u$ isolieren. Mit dieser Methode gelang es zu beweisen, daß nicht $30 \%$ des Serum- $\mathrm{T}_{4}$, sondern nur etwa $15 \%$ an TBPA gebunden sind (3). Nach der gleichen Methode, jedoch ohne Zusatz von radioaktivem $\mathrm{T}_{4}$ zum Serum, fanden wir bei gesunden Personen zwischen 19 und 21\% Bindung des Gesamt- $T_{4}$ an TBPA (unveröffentlichte Ergebnisse).

In der vorliegenden Arbeit wurde dạs Bindungsvermögen von Schilddrüsenhormonen an Lipoproteine unter physiologischen Bedingungen mit Hilfe immunochemischer Methoden untersucht. Der von Lipoproteinen gebundene Hormonanteil betrug unter den gewählten Bedingungen weniger als $0,4 \%$.

\section{Material und Methoden}

Die Analyse der Blutproben, die 12 Stdn. nach der letzten Nahrungsaufnahme abgenommen wurden, erfolgte innerhalb von 24 Stdn. Es wurden Seren von Euthyreoten, Hyperthyreoten und Hypothyreoten eingesetzt. In vivo radioaktiv markierte Seren wurden 48 Stdn. nach Gabe von 3-100 mC ${ }^{131}$ J (Radio-Jodtherapie) abgenommen.

Reine Antikörper gegen $\beta$-Lipoproteine wurden nach G. KostNER und A. HolASEK (19) gewonnen. Die für die vorliegenden Untersuchungen verwendeten reinen Antikörper stammten vom Pferd und wurden durch Druckdialyse gegen isotone $\mathrm{NaCl}$-Lösung auf eine Konzentration von etwa $8 \%$ gebracht. Mit diesem Antikörper wurde auch ein Immunoadsorber hergestellt (20). Nach einem etwas modifizierten Verfahren wurde reiner Antikörper gegen Human- $\alpha$-Lipoproteine vom Kaninchen präpariert (in Vorbereitung). 
Die Isolierung reiner Lipoproteine erfolgte nach Einstellen der Dichte des Serums mit festem $\mathrm{KBr}$ auf die Werte 1,063 und 1,21 in einer präparativen Ultrazentrifuge (Beckman L 4). Very low density lipoproteins (VLDL) wurden bei der Eigendichte des Serums durch Flotation gewonnen. Nach dem Zentrifugieren wurden die Lipoproteinlösungen gegen isotone $\mathrm{NaCl}$-Lösung vom $\mathrm{pH} 7,4$ dialysiert und gleich für die Untersuchung eingesetzt.

Nach der Gewinnung von Serumlipiden durch Extraktion von $20 \mathrm{ml}$ gepooltem Humanserum nach Folch (21) wurde das Lösungsmittel unter Vakuum abgedampft und die Lipide in Krebs-Ringerlösung mit einem Ultraschallgerät emulgiert. Dieses Gemisch wurde mit $L_{-}-T_{4}{ }^{-131} \mathrm{~J}$ inkubiert. Anschließend wurden die Lipide von der Krebs-Ringerlösung in der Ultrazentrifuge mit einem SW-Rotor bei $2 \cdot 10^{8} \mathrm{~g} / \mathrm{Min}$. abgetrennt.

Radioaktives $\mathrm{L}_{-} \mathrm{T}_{\mathbf{4}}{ }^{-131} \mathrm{~J}$ mit einer spezifischen Aktivität von $20-50 \mathrm{mC} / \mathrm{mg}$, das vor dem Einsatz papierchromatographisch gereinigt wurde, stammte vom Radiochemical Centre Amersham.

Um die Seren und Lipoproteinlösungen in vitro radioaktiv zu markieren, wurden zu je $20 \mathrm{~m} /$ Lösung $4 \mu \mathrm{C}_{L}-\mathrm{T}_{4}{ }_{-131} \mathrm{~J}$ zugesetzt. Diese Mischung blieb vor der Analyse 3 Stdn. bei Raumtemperatur und 12 Stdn. bei $4^{\circ}$ stehen.

Die Aktivitäten wurden mit dem Zählgerät Frieseke und Hoepfner 49 A Grundsonde FH 421AS, Bohrlochscintillationskristall Z 11 in Verbindung mit einem 100 Kanal-Gamma-Spectrometer, TMC Gammascope II in Einkanalschaltung $(0,36 \mathrm{MeV})$ gemessen. Zur Wahrung der Geometrie wurden alle Proben in identischen Küvetten mit gleichem Volumen $(4 \mathrm{ml})$ vermessen.

Die Werte des eiweißgebundenen Jods (PBI-Werte) wurden in einer Apparatur zur automatischen Jodbestimmung (22) ermittelt.

Alle zur Analyse verwendeten Chemikalien waren p. A. Reagenzien der Firma E. Merck.

\section{Arbeitstechnik}

Die Lipoproteine wurden im Åquivalenzpunkt, der von jedem Serum und von jeder Lipoproteinlösung nach HEIDELbERger (23) bestimmt wurde, präzipitiert. $\mathrm{Zu}$ je $1 \mathrm{ml}$ Probe wurde reine Antikörperlösung (etwa $15 \mu l$ ) zugesetzt und die Mischung 2 Stdn. bei Raumtemperatur und $14 \mathrm{Stdn}$. bei $4^{\circ}$ stehen gelassen. Anschließend wurde zentrifugiert und das Präzipitat 2- bzw. $4 \mathrm{mal} \mathrm{mit} \mathrm{je} 3 \mathrm{ml}$ isotoner Natriumchloridlösung, deren $\mathrm{pH}$-Wert mit $\mathrm{NaOH}$ auf 7,4 eingestellt wurde, gewaschen und in $0,1 \mathrm{~N}$ $\mathrm{NaOH}$ (auf $1 \mathrm{~m} /$ ) gelöst. Von der ursprünglichen Probe, dem Uberstand nach dem Zentrifugieren und dem gelösten Immunpräzipitat wurden Aktivitätsmessungen von je $4 \mathrm{~m} /$ Probe und PBI-Bestimmungen von je $0,2 \mathrm{~m} l$ Probe durchgeführt.

Die Immunoadsorption der low density lipoproteins (LDL) + VLDL an mit Antikörper beladene Bromacetylcellulose (20) erfolgte, indem zu jeder Probe soviel Immunoadsorber zugesetzt wurde, daß bei anschließender Ringprobe keine positive Reaktion erhalten wurde.

Zur Adsorption der Gesamt-Lipoproteine an Aerosil (24) wurde je $1 \mathrm{~m} l$ Serum bzw. Lipoproteinlösung mit $20 \mathrm{mg}$ Aerosil versetzt und $6 \mathrm{Stdn}$. bei $45^{\circ}$ gerührt. Anschließend wurde zentrifugiert, der Niederschlag mit isotoner $\mathrm{NaCl}-\mathrm{L} .0 ̈$ sung vom pH 7,4 (4 mal je $3 \mathrm{~m} /$ ) gewaschen und die Lipoproteine mit 40proz. NaCl-Lösung in $0,1 \mathrm{~N} \mathrm{NaOH}$ desorbicrt.

$\mathrm{Zu} 1 \mathrm{~m} /$ reiner Lipoproteinlösung wurden $0,5 \mathrm{~m} /$ bzw. $0,25 \mathrm{~m} /$ 60proz. Trichloressigsäure zugesetzt. Nach dem Zentrifugieren wurde die Aktivität der überstehenden Lösung bestimmt.

\section{Ergebnisse}

\section{Ermittlung der Bindung von Scbilddrisenbormonen an Lipo- proteine durch PBI-Bestimmungen}

Tabelle 1 zeigt die PBI-Werte von 10 verschiedenen Seren vor und nach der Fällung der Lipoproteine mit spezifischen Antikörpern bzw. Adsorption mit Aerosil.

Tab. 1

PBI-Werte in $\mu \mathrm{g} / 100 \mathrm{ml}$ von Humanseren vor und nach der Fällung der VLDL, LDL und HDL mit spezifischen Antikörpern und Adsorption der Gesamtlipoproteine an Aerosil

\begin{tabular}{|c|c|c|c|c|}
\hline Serum Nr. & $\begin{array}{c}\text { Ausgangs- } \\
\text { serum }\end{array}$ & $\begin{array}{l}\text { PBI in } \mu \\
\text { Serum ohne } \\
\text { VLDL und } \\
\text { LDL } \\
\text { (Fällung mit } \\
\text { AK) }\end{array}$ & $\begin{array}{l}\text { Serum ohne } \\
\text { HDL } \\
\text { (Fällung mit } \\
\text { AK) }\end{array}$ & $\begin{array}{c}\text { Serum ohne } \\
\text { Lipopro- } \\
\text { teine } \\
\text { (Adsorption } \\
\text { an Aerosil) }\end{array}$ \\
\hline 1 St. L. & 2,1 & 2,1 & 2,2 & 2,1 \\
\hline 2 K. G. & 2,8 & 2,8 & 2,8 & 2,7 \\
\hline 3 N. A. & 4,6 & 4,5 & 4,6 & 4,7 \\
\hline 4 W. A. & 5,6 & 5,6 & 5,6 & 5,6 \\
\hline 5 P. L. & 6,5 & 6,4 & 6,5 & 6,4 \\
\hline 6 H. B. & 6,8 & 6,9 & 6,8 & 6,9 \\
\hline 7 A. H. & 7,2 & 7,3 & 7,2 & 7,3 \\
\hline 8 K. K. & 10,5 & 10,4 & 10,4 & 10,6 \\
\hline 9 M. S. & 16,7 & 16,7 & 16,7 & 16,7 \\
\hline 10 I. Sch. & 18,0 & 18,0 & 18,1 & 18,0 \\
\hline
\end{tabular}

Die Verdünnung der Seren mit Antikörperlösung ist in den Werten der Tabelle berücksichtigt. Alle Zahlen stellen Mittelwerte aus 3 Bestimmungen dar. Die Abweichung der Serum-PBI-Werte vor und nach der Fällung der Lipoproteine liegt innerhalb der Fehlergrenze der Methode. Vom gefällten Präzipitat wurde keine PBI-Bestimmung durchgeführt, da PBI-Werte unter $0,5 \mu \mathrm{g} / 100 \mathrm{~m} l$ mit dieser Methode nur ungenau bestimmt werden können.

\section{Radioaktivitätsmessungen an in vivo markierten Seren}

Die Analysenergebnisse der radioaktiven in vivo markierten Seren sind in Tabelle 2 und 3 zusammengefaßt. Im Immunpräzipitat der Lipoproteine sind nach 2 mali-

Tab. 2

Aktivität von in vivo radioaktiv markierten Humanseren vor und nach der Fällung der Lipoproteine mit spezifischen Antikörpern bzw. Adsorption der Gesamtlipoproteine an Aerosil

\begin{tabular}{|c|c|c|c|c|c|c|c|}
\hline Serum Nr. & $\begin{array}{l}\text { Verabreichte } \\
\text { Menge }{ }^{121} \mathrm{~J} \text { in } \\
\mathrm{mC}\end{array}$ & $\begin{array}{c}\text { PBI in } \\
\mu \mathrm{g} / 100 \mathrm{ml}\end{array}$ & $\begin{array}{c}\text { Ausgangs- } \\
\text { serum }\end{array}$ & $\begin{array}{l}\text { Aktivität } \\
\text { Serum nach } \\
\text { Fällung d. } \\
\text { LDL u. VLDL } \\
\text { mit Antikörpern }\end{array}$ & $\begin{array}{l}\text { on } 1 \mathrm{ml} \text { Serum i } \\
\text { Serum nach } \\
\text { Adsorption d. } \\
\text { LDL u. VLDL } \\
\text { mit Immuno- } \\
\text { adsorber }\end{array}$ & $\begin{array}{l}\text { mp./Min. } \\
\text { Serum nach } \\
\text { Fällung d. } \\
\text { HDL mit } \\
\text { Antikörpern }\end{array}$ & $\begin{array}{l}\text { Überstand nach } \\
\text { Adsorption d. } \\
\text { Lipoproteine mit } \\
\text { Aerosil }\end{array}$ \\
\hline $11 \mathrm{H} . \mathrm{H}$. & 3,2 & 16,1 & 28240 & 28420 & 28100 & 28310 & 28120 \\
\hline 12 B. Sch. & 4,4 & 12,4 & 31780 & 31490 & 31610 & 31980 & 31810 \\
\hline 13 L. M. & 5,0 & 14,1 & 35200 & 35120 & 35280 & 35470 & 35370 \\
\hline 14 J. G. & 100,0 & 1,2 & 40030 & 40370 & 40120 & 39690 & 40130 \\
\hline 15 H. F. & 100,0 & 3,2 & 112120 & 112490 & 111970 & 112360 & 112300 \\
\hline
\end{tabular}


Tab. 3

Radioaktivität der VLDL, LDL und HDL nach der Fällung mit spezifischen Antikörpern bzw. Adsorption mit Aerosil aus in vivo radioaktiv markierten Seren

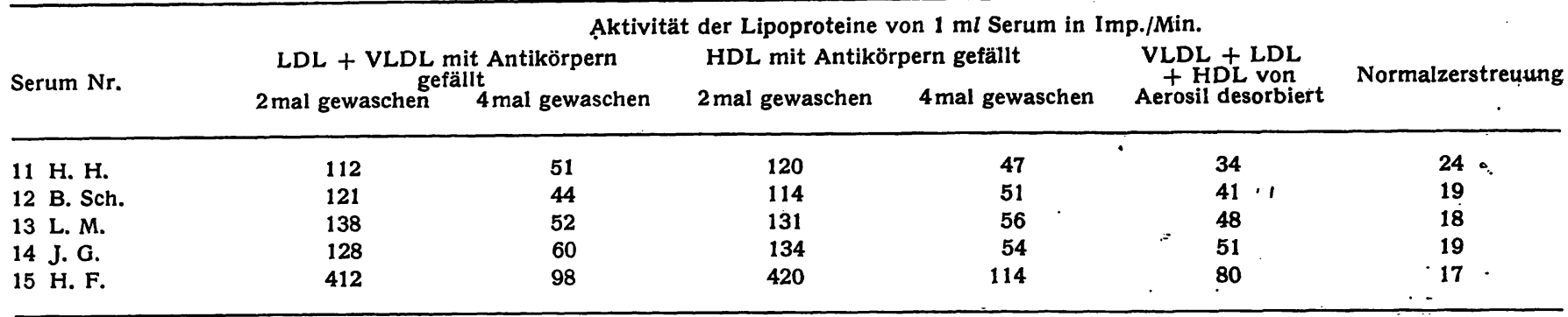

Tab. 4

Lösung von $T_{4}-181 \mathrm{~J}$ in VLDL, LDL und HDL: Nach der Inkubation der reinen Lipoproteine mit $\mathrm{T}_{4}^{-131} \mathrm{~J}$ wurde eine Fällung mit Antikörpern, eine Adsorption an Aerosil und eine Fällung mit Trichloressigsäure durchgeführt und die Aktivität bestimmt

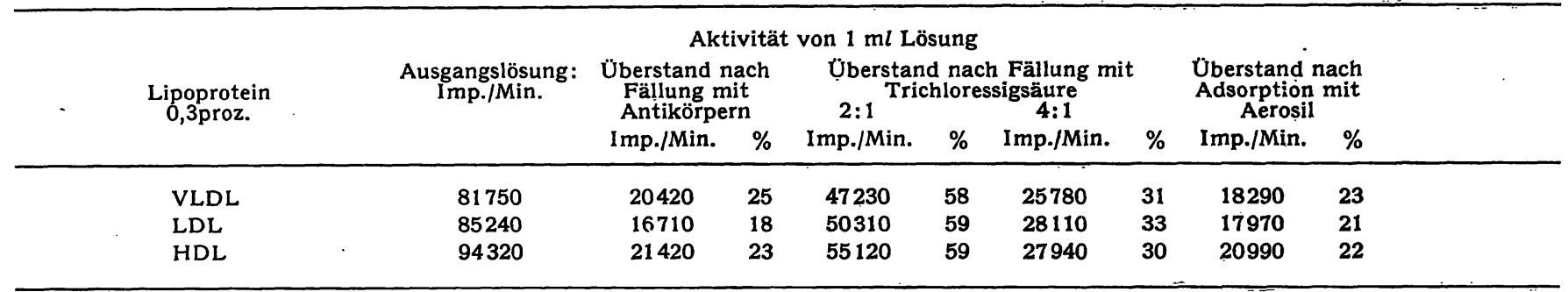

gem Waschen etwa $0,3 \%$ und nach 4 maligem Waschen nur mehr $>0,1 \%$ der Gesamtaktivität vorhanden. Die Variation der Zählraten der Seren vor und nach der Entfernung der Lipoproteine liegt innerhalb der Fehlergrenze der Aktivitätsmessung.

\section{Ergebnisse der in vitro mit $\mathrm{T}_{4}{ }^{131} \mathrm{~J}$ markierten Seren}

Um den Unterschied zwischen in vivo und in vitro Versuchen festzustellen, wurden Humanseren mit $\mathrm{T}_{\mathbf{4}^{\mathbf{1}}}{ }^{\mathbf{1 3 1}} \mathrm{J}$ versetzt und nach Inkubation die gleichen Versuche wie oben durchgeführt. Die Werte zeigten praktisch keine Unterschiede. Nach 4 maligem Waschen des Immunpräzipitates war weniger als $0,1 \%$ der Aktivität in den Lipoproteinen zu finden. Selbst nach Absättigung des TBG und TBPA durch Zusatz von inaktivem $T_{4}$ $(0,05 \mathrm{mg} / \mathrm{ml})$ und anschließender Inkubation mit $\mathrm{T}_{4}{ }^{-131} \mathrm{~J}$ konnte keine höhere Aktivität in den Lipoproteinen gemessen werden.

\section{In vitro $V$ ersucbe mit reinen Lipoproteinen}

Nach der Inkubation reiner VLDL, LDL und high density lipoproteins (HDL) einer Konzentration von etwa $0,3 \%$ mit $\mathrm{T}_{4^{-131} \mathrm{~J}}$ wurden die Lipoproteine mit reinem Antikörper bzw. 60proz. Trichloressigsäure gefällt. Tabelle 4 enthält die auf diese Weise gewonnenen Ergebnisse. Die Aktivitätsverteilung zwischen Lipoproteinpräzipitat und Überstand hängt offenbar von der Art der Fällung bzw. Adsorption ab und bewegt sich zwischen 20 und $80 \%$ Bindung des Gesamt- $\mathrm{T}_{4}$ an Lipoproteine.

Aktivitätsverteilung von $\mathrm{T}_{\mathbf{4}^{-131} \mathrm{~J}}$ qwischen wäßriger und Lipidphase einer Emulsion von Serumlipiden in Krebs-Ringerlösung

Um zu untersuchen, ob der Proteinanteil der Lipoproteine eine Rolle bei der Bindung von $T_{4}$ spielt oder ob die Aktivität der Lipoproteine nur durch Gleichgewichtseinstellung auf Grund der Nernst'schen Verteilung hervorgerufen wird, wurde die Verteilung von $\mathrm{T}_{4}{ }^{131} \mathrm{~J}$ zwischen Wasser- und Lipidphase untersucht. Aus Tabelle 5 kann entnommen werden, daß etwa $65 \%$ der Radioaktivität in der Lipidphase vorhanden war.

Tab. 5

Verteilung von $T_{4^{-132} \mathrm{~J}}$ zwischen wäßr. und Lipidphase nach Inkubation einer Lipidemulsion in Krebs-Ringerlösung

\begin{tabular}{ccccc}
\hline Lösung & $\begin{array}{c}\text { Lipide } \\
\text { mg/100 ml }\end{array}$ & $\begin{array}{c}\text { Aktivität von } \\
\text { I ml in } \\
\text { Imp./Min. }\end{array}$ & $\begin{array}{c}\text { Aktivität der wäßr. } \\
\text { Phase nach Zentri- } \\
\text { fugieren } \\
\text { Imp./Min. }\end{array}$ & $\%$ \\
\hline A & 200 & 91280 & 31240 & 34 \\
B & 200 & 78450 & 27400 & 35 \\
C & 200 & 74230 & 27580 & 37 \\
\hline
\end{tabular}

\section{Diskussion}

Die Untersuchung der Bindung von Thyroxin an Serumproteine mit Hilfe der Papier-(16), Acetatfolien-(25), Stärkekegel- und Agargelektrophorese (16) sowie Polyacrylamidgelelektrophorese (26) ergab, daß neben einigen unbekannten Proteinen TBG, TBPA und TBA die Hauptmenge der jodhaltigen Schilddrüsenhormone im Serum transportieren. Neben den individuellen Schwankungen, hervorgerufen durch Alter. und Geschlecht (27), Schwangerschaft (28) und verschiedene Krankheiten (29) ist, wie erwähnt, die qualitative und quantitative Verteilung der Schilddrüsenhormone unter den Serumproteinen stark von den Versuchsbedingungen abhängig. Daher ist es notwendig, die zu untersuchenden Proteine möglichst rasch und schonend $\mathrm{zu}$ isolieren. Diese Bedingungen werden praktisch nur von immunochemischen Methoden erfüllt. Bei Zusatz von radioaktivem $T_{4}$ und anschließender Elektrophorese (16) können schon deshalb keine genauen 
Ergebnisse der quantitativen Verteilung erhalten werden, da einerseits im Serum neben $\mathrm{T}_{4}$ auch Trijodthyronin, Dijodthyronin und Monojodthyronin vorhanden ist (30), andererseits eine geringe Menge Thyreoglobulin vorkommt, welches beim Menschen die gleiche elektrophoretische Wanderungsgeschwindigkeit besitzt wie TBG (31). Da gerade mit Hilfe immunochemischer Methoden, insbesondere mit der Immunoelektrophorese, gefunden wurde, daß die Serumlipoproteine Thyroxin zu binden vermögen ( $6-10)$, andererseits die Immunoadsorption von INGBAR (3) als die zuverlässigste Methode zur Bestimmung der quantitativen Verteilung der Schilddrüsenhormone unter den Serumproteinen beschrieben ist, sollte mit dieser Methode die Rolle der Lipoproteine als Träger der Schilddrüsenhormone untersucht werden.

Die Ergebnisse dieser Arbeit zeigen, daß weder $\alpha$-Lipoproteine (HDL), noch $\beta$-Lipoproteine (LDL), noch prae- $\beta$-Lipoproteine (VLDL) in der Lage sind, in vivo oder in vitro im Gemisch mit den übrigen Serumproteinen nennenswerte Mengen von Schilddrüsenhormonen zu binden. Selbst nach Absättigung der freien Bindungsstellen des TBPA und TBG mit Thyroxin hat das Albumin für $T_{4}$ offenbar noch eine viel größere Bindungsfähigkeit als die Lipoproteine. Die Absättigung erfolgte durch Zusatz von inaktivem $T_{4}$ in zweifachem molarem Úberschuß unter der Annahme einer 1:1 Bindung von $\mathrm{T}_{4}$ an diese Proteine und einer Konzentration des TBPA von $30 \mathrm{mg} / 100 \mathrm{ml}$ Serum und der des TBG von 1-2 $\mathrm{mg} / 100 \mathrm{ml}$ Serum (32). In Abwesenheit aller anderen Serumproteine besitzen reine LDL, VLDL und HDL die Fähigkeit, eine beträchtliche Menge (etwa 80\%, wenn die Versuche mit Proteinkonzentrationen vorgenommen werden, die denen im Serum entsprechen) des zugesetzten $\mathrm{T}_{4}^{-131} \mathrm{~J}$ aufzunehmen. Wie die Versuche mit den Lipidemulsionen zeigten, dürfte es sich dabei um keine spezifische Bindung an die Apoproteine sondern um eine Lösung im Lipidanteil auf Grund der Nernst'schen Verteilung handeln. Mit Hilfe dieser Versuche wurde gefunden, daß sich lediglich etwa $65 \%$ des $z u-$ gesetzten $\mathrm{T}_{4}$ in der Lipidphase befanden, also weniger als in den Lipoproteinen. Dies hat seinen Grund darin, daß beim Emulgieren der Serumlipide in Krebs-Ringerlösung Lipidmizellen entstehen, die eine Lösungsfähigkeit für $\mathrm{T}_{4}$ besitzen. Diese Lipidmizellen wurden bei der präparativen Abtrennung der Lipide in der Ultrazentrifuge nicht aus der wäßrigen Phase entfernt.

Bei der Fällung der Lipoproteine mit Trichloressigsäure (Tab. 4) zeigte sich, daß bei Zusatz der doppelten Menge dieses Reagenz viel mehr $T_{4}$ in der wäßrigen Phase zu finden war als bei Zusatz der einfachen Menge. Hier kommt es offenbar zu einer Verschiebung des Verteilungskoeffizienten. Inaktive Seren (Tab. 1), bei denen die PBI-Werte der Lipoproteinpräzipitate nicht ermittelt werden konnten, wurden untersucht, da radioaktive Humanseren nur von Patienten mit Schilddrüsenstörungen zugänglich waren. Die Ergebnisse waren jedoch in beiden Fällen praktisch identisch.

Von INGBAR (3) wurde bewiesen, daß Antikörper die Bindung von $T_{4}$ an TBPA nicht beeinträchtigen. Wie die Adsorptionsversuche mit Aerosil zeigten, kann das gleiche von den Lipoproteinen angenommen werden.

Nach 2 maligem Waschen war im Präzipitat eine, wenn auch sehr geringe, Restaktivität zu finden. Da im Serum neben dem proteingebundenen Thyroxin auch ein kleiner Teil freies $T_{3}$ und $T_{4}$ vorhanden ist $\left(T_{4}: 100\right.$ $\mu \mathrm{Mol} / 100 \mathrm{~g}$ Serum und $\mathrm{T}_{3}: 100 \mu \mathrm{Mol} / 100 \mathrm{~g}$ Serum (33)), dürfte sich eine geringe Menge im Gleichgewicht in den Lipoproteinen lösen. Dieser Teil genügt jedoch nicht, die Lipoproteinbanden nach Immunoelektrophorese und Autoradiographie von in vivo radioaktiv markierten Seren sichtbar zu machen. Die Radioaktivität dieser Seren reichte auch nicht aus, um damit nach einer Immunoelektrophorese ein befriedigendes Autoradiogramm der $\mathrm{T}_{4}$-bindenden Proteine $\mathrm{zu}$ erhalten. Die Fähigkeit der isolierten Lipoproteine, $T_{4} \mathrm{zu}$ lösen, macht es verständlich, daß nach der von $R$. W. LrGHTFoor (34) vorgeschlagenen Methode, bei der nach Beendigung der Immunodiffusion und dem Auswaschen der nicht präzipitierten Proteine, aktives $\mathrm{T}_{4}$ in den Antiserumschlitz gefüllt wird, Aktivität in den Lipoproteinbanden zu finden ist. Bei der Ausführung der Immunoelektrophoresen mit Seren, welche in vivo mit $\mathrm{T}_{4}{ }^{-131} \mathrm{~J}$ versetzt wurden, zeigte sich ebenso eine Schwärzung der Lipoproteinbanden im Autoradiogramm (6). Da Phospholipide, die einen Hauptbestandteil des Lipidanteils der Lipoproteine ausmachen, in der Lage sind, freies Jodid zu binden (35), ist es möglich, daß bei Verwendung unreiner $\mathrm{T}_{\mathbf{4}}$-Präparate Artefakte entstehen.

Herrn Dr. K. MüLler, Assistent am Institut für Allgemeine Chemie, Mikro- und Radiochemie der Technischen Hochschule Graz, danken wir für die Ausführung der Radioaktivitätsbestimmungen und Herrn Doz. Dr. O. Ener, Oberarzt an der Medizinischen Universitätsklinik Graz, für die freundliche Unterstützung und Bereitstellung des Untersuchungsmaterials. Außerdem danken wir dem Fonds zur Förderung der wissenschaftlichen Forschung für die Ubberlassung einer Ultrazentrifuge.

\section{Literatur}

1. Giorgio, M., A. Nrchoras und M. Tabacknick, J. biol. Chemistry 243, 2247 (1968). - 2. HollaNDER, CH. S., V. V. ODAK, T. E. Prout und S. P. Asper, J. Clin. Endocr., Springfield 22, 617 (1962). 3. Worber, K. A. und S. H. Ingbar, J. Clin. Invest. 47, 1710 (1968). - 4. Oppenherimer, J. H. und G. Bernstein, Current Topics in Thyroid Research, Acad. Press. N. Y. 674 (1965). - 5. TATA, J. R., Clin. chim. Actn, Amsterdam 6, 597 (1961). - 6.
Miya, K., K. F. H. Age und Y. Kumafiara, Clin. chim. Acta, Amsterdam, 23, 341 (1968). - 7. Gerhards H. J., K. Joseph und E. H. Graud, Atompraxis 14, 21 (1968). - 8. Hollander, Ch. S., B. H. Latimbr, T. E. Prout, D. H. Lockwood und S. P. Asper. Metabolism, Baltimore 12, 45 (1963). - 9. AlY, F. W. und K. H. Grrlich in PeErbrs H. (Ed.) Protides of the Biological Fluids 8 th Colloquium, Brugges 1960, Elsevicr Amsterdam 152 (1961). - 
10. Clausen, J. und T. Munkner in Peeters H. (Ed.) Protides of the Biological Fluids, Brugges 1960, Elsevier Amsterdam 147 (1961). - 11. Lohss, F. und E. Kallel in Peeters H. (Ed.) Protides of the Biological Fluids, Brugges 1960, Elsevier Amsterdam 142 (1961). - 12. Lightfoot, R. W. und C. L. Christian, J. Clin. Endocr. Springfield, 26, 305 (1966). - 13. Rosbins, J. und J. E. RaLl, Physiol. Rev. Baltimore 40, 414 (1960). - 14. Lutz, J. H. und R. I. GregermaN, J. Clin. Endocr. Springfield 29, 487 (1969). 15. Squaf, R. M., K. Marinetz und J. H. Oppenheimer, Proc. Soc. Exper. Biol. Med. N. Y. 113, 837 (1963). - 16. INGBAR, S. H. und L. E. BRAVERMAN, in Endocrinologica Experimentalis, S. 253 Publishing House Acad. Sci. Bratislava, (1966). - 17. TAKashr Yamanda, A. und E. Jones, Endocrinology 82, 47 (1968). - 18. INGBAR, S. H., Endocrinology 63, 256 (1958). - 19. KOSTNER, G. und A. Holasek, Biochim. biophysica Acta Amsterdam 188, 157 (1969). - 20. Kostner, G. und A. Holasek, Lipids, Arbeit zur Publikation eingereicht. - 21. FolCH, J. M., G. H. S. LeES und S. Stanlex, J. biol. Chemistry 226, 497 (1957). - 22. Knapp, G. und H. Spitzy, Talanta, im Druck. - 23. Herdelberger, M. und
E. A. KABAT, J. Exper. Med. 67, 181 (1938). - 24. STEPHAN, W., L. Roka, diese Z. 6, 186 (1968). - 25. Lauvinay, M. P., Canad. J. Biochem. 44, 1657 (1966). - 26. RoBierts, R. C. und T. F. Nrcolar, Clin. Chem. Nèw York 15, 367 (1969). - 27. Braverman, L. E., B.E. Foster und S. H. IngBar, J. Clin. Endocr., Springfield 27, 227 (1967). - 28. FrIEdrICH, Z., E. T. RIPPMANN und V. Kaufmani, Schweiz. med. Wschr. 99/23, 833 (1969). - 29. Negoesco, J. und A. Constantinesco, Rev. Roum. Endocrin. 5, 55 (1968). - 30. WeLLBY, M. L. und M. W. O'Hallovan, Biochem. J. 112, 543 (1969). - 31. Torriglani, G., D. Doriach und I. M. Rorrt, J. Clin. Endocr., Springfield 29, 305 (1969). - 32. Schultze, H.F. und J.F. Heremans, Molecular Biology of Human Proteins S. 175, Elsevier Amsterdam (1966). - 33. HrilMAN, G., Biosynthese und Stoffwechselwirkungen d. Schilddrüsenhormone, S. 43, Thieme Verlag Stuttgart (1961). - 34. LigHrFoor; R. W., C. L. Christian, J. Clin. Endocr., Springfield 26, 305 (1966). - 35. Phopesherarkar, G. A. und M. Y. Mandrix, Proc. Soc. Exper. Biol. Med. N. Y. 192, 571 (1968).

Dr. Gerhard Kostner Inst. f. physiologische Chemie d. Universität Graz Universitätsplatz 2 A-8010 Graz 